\title{
Is Breast Cancer the Same Disease in Asian and Western Countries?
}

\author{
Stanley P. L. Leong $\cdot$ Zhen-Zhou Shen $\cdot$ Tse-Jia Liu • \\ Gaurav Agarwal · Tomoo Tajima - Nam-Sun Paik - Kerstin Sandelin • \\ Anna Derossis $\cdot$ Hiram Cody $\cdot$ William D. Foulkes
}

Published online: 7 July 2010

(c) The Author(s) 2010. This article is published with open access at Springerlink.com

\begin{abstract}
A mini-symposium was held in Montreal, Canada, at the International Surgical Week for the Breast Surgical International in 2007 addressing the question whether breast cancer is the same disease in Asian and Western countries. Numerous investigators from Asian and Western countries presented the epidemiologic and clinical outcome data of women with breast cancer. Although there are significant similarities, the striking difference is that the peak age for breast cancer is between 40 and 50 years in the Asian countries, whereas the peak age in the Western countries is between 60 and 70 years. Also, the incidence of breast cancer in Asia is rising and is associated with increased mortality. In the West, although the incidence is increasing, the mortality rate is definitely decreasing. Future prospective data collection from Asian and Western countries may provide further interesting epidemiologic
\end{abstract}

S. P. L. Leong $(\bowtie)$

Department of Surgery, California Pacific Medical Center and Sutter Pacific Medical Foundation, 2340 Clay Street, 2nd Floor, San Francisco, CA 94115, USA

e-mail: leongsx@cpmcri.org

\section{Z.-Z. Shen}

Department of Surgery, Fudan University,

Shanghai, People's Republic of China

T.-J. Liu

Department of Surgery, Taipei Medical University,

Wanfang Medical Center, Taipei, Taiwan

\section{G. Agarwal}

Department of Endocrine and Breast Surgery, Sanjay Gupta Postgraduate Institute of Medical Sciences, (SGPGIMS),

Lucknow 226014, India

T. Tajima

Department of Surgery, Tokai University, Tokyo, Japan and outcome data regarding the outcome of women with breast cancer from Asian and Western countries.

Background Whether breast cancer is the same disease in Asian and Western countries was the topic of a 2007 Breast Surgery International symposium at International Surgical Week.

Methods Participating investigators from China, Taiwan, India, Japan, South Korea, Sweden, Canada, and the United States were asked beforehand to provide data on the epidemiology and treatment outcome of women in their countries.

Results Comparisons of the epidemiologic and clinical outcome data of women with breast cancer showed significant similarities, but the striking difference is that the peak age is between 40 and 50 years in Asian countries, but is between 60 and 70 years in Western countries.

\section{N.-S. Paik}

Department of Surgical Oncology, Korea Cancer Center Hospital, Seoul, South Korea

K. Sandelin

Deparment of Molecular Medicine and Surgery, Karolinska Institutet, Stockholm, Sweden

A. Derossis

Department of Surgery, McGill University, Montreal, Canada

H. Cody

Department of Surgery, Memorial Sloan-Kettering Hospital,

New York, NY, USA

W. D. Foulkes

Department of Medicine, McGill University, Montreal,

Canada 
The incidence of breast cancer in Asia is rising and is associated with increased mortality. In the West, although the incidence is also increasing, the mortality rate is definitely decreasing.

Discussion Future prospective data collection from Asian and Western countries may provide further interesting epidemiologic and outcome data regarding the outcome of women with breast cancer from Asian and Western countries.

\section{Introduction}

Although the incidence of breast cancer has increased globally over the last several decades [1-3], the greatest increase has been in Asian countries [4]. In Asia, breast cancer incidence peaks among women in their forties [4], whereas in the United States and Europe, it peaks among women in their sixties. This variation in incidence may be due to multiple factors, including geographic variation, racial/ethnic background, genetic variation, lifestyle, environmental factors, socioeconomic status, the presence of known risk factors, utilization of screening mammography, stage of disease at diagnosis, and the availability of appropriate care [2]. Whether these discrepancies indicate that breast cancer is the same disease in Asia as it is in Western countries was the topic of a Breast Surgery International (BSI) symposium attended by surgeons from many countries during the 2007 Montreal International Surgical Week. To discern whether breast cancer characteristics other than incidence differ between Asian and Western countries, breast cancer experts from China, Taiwan, India, Japan, South Korea, Europe, Canada, and the United States summarized their experiences in this report.

\section{Materials and methods}

Experts from the various countries who participated in the symposium were asked beforehand to complete a table containing multiple questions (Table 1). Participating investigators represented China, Taiwan, India, Japan, South Korea, Sweden, Canada, and the United States. Each investigator was also asked to write a summary of how the data requested in the table were collected.

\section{Results}

Data for every question were not available for all countries participating in the symposium. The following summaries are presented by the representative from each country.
China

\section{Data collection and incidence estimates}

There is a lack of countrywide effort to collect data on breast cancer in China. Most data have been obtained from cities and suburban areas such as Shanghai, Beijing, Harbin, Wuhan, and Qigong. The data from these areas are evaluated by the Statistics Center within the Health Ministry of the People's Republic of China. Shanghai's Municipal Center for Disease Control and Prevention (CDCP) plays an active role in cancer surveillance. Most data published in China come from the Shanghai CDCP and Fudan University Cancer Hospital, or from articles published in China. Data from Fudan University are derived from a database established by the Department of Breast Surgery, which has collected more than 10,000 records of patients, including detailed clinical and pathologic data with follow-up information.

Incidence estimates vary for China. China's Health Ministry data indicate an incidence of 24/100,000. According to global cancer statistics for the year 2002 [5], the age-standardized incidence for breast cancer is 18.7/ 100,000 in China. However, in Shanghai, the incidence is considerably higher, at 61/100,000; after age standardization, the incidence is 37/100,000 (2003 data from Shanghai CDCP). Data from Shanghai's CDCP showed that in 2003, the age distribution of breast cancer patients in China was different from that in the West, with a peak among women 45-50 years old.

\section{Screening and methods of detection}

Data from Fudan University Cancer Hospital show that breast self-examination is recommended to cancer patients and healthy women. It is recommended that women over the age of 40 have a mammogram each year. The breast cancer screening program is carried out well in urban Shanghai.

\section{AJCC stage of initial presentation}

In Fudan Cancer Hospital, stage II is the most common stage of newly diagnosed breast cancer, accounting for about $50 \%$ of cases. The percentage of newly diagnosed stage I breast cancers is up to $20 \%$ and still increasing, whereas the percentage of stage III breast cancers has decreased from $40 \%$ in the 1970 s to $20 \%$ in 1990 s.

\section{Treatment}

In China, surgical treatment for breast cancer varies in different provinces and areas, and even varies within the 


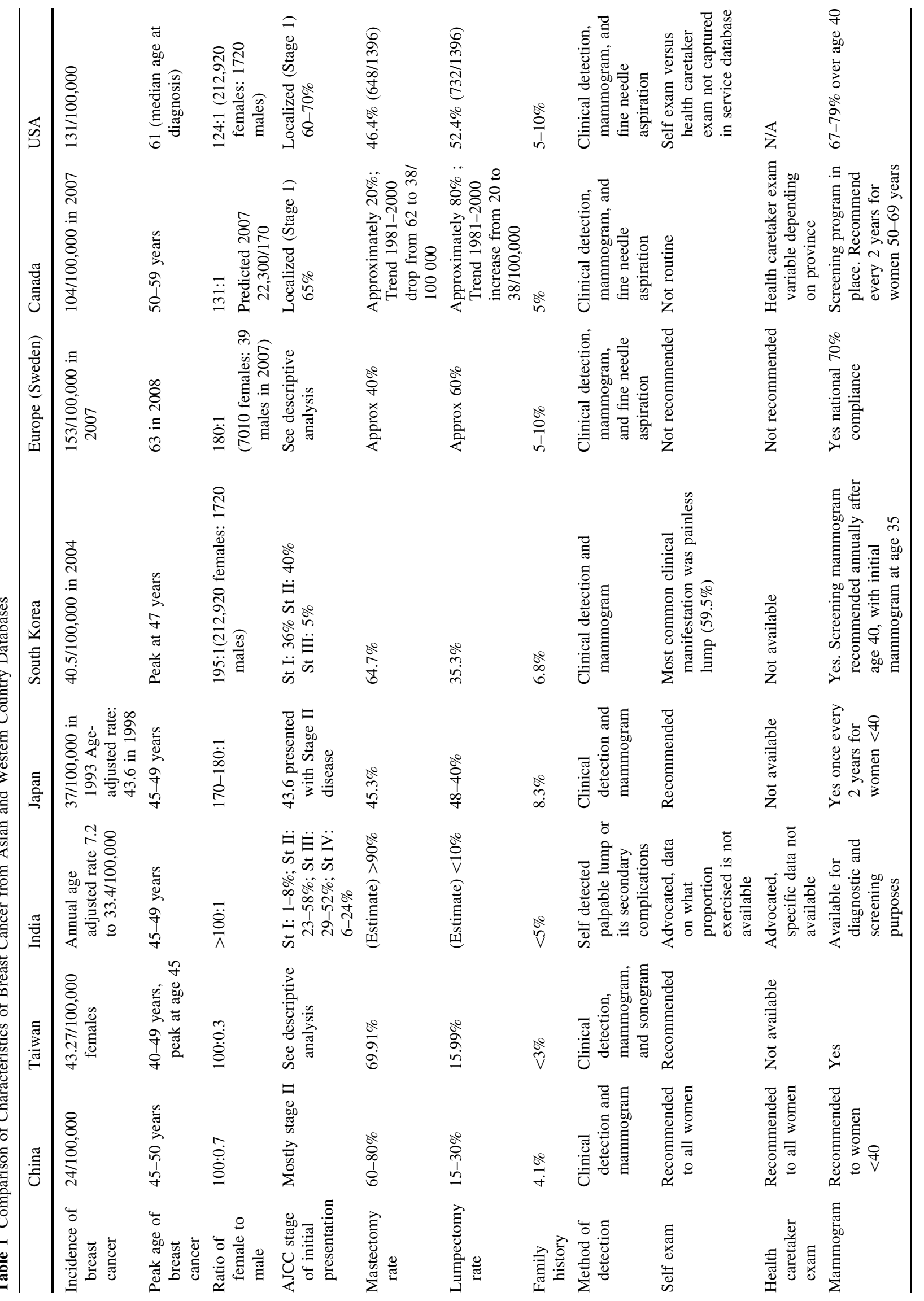


World J Surg (2010) 34:2308-2324

2311

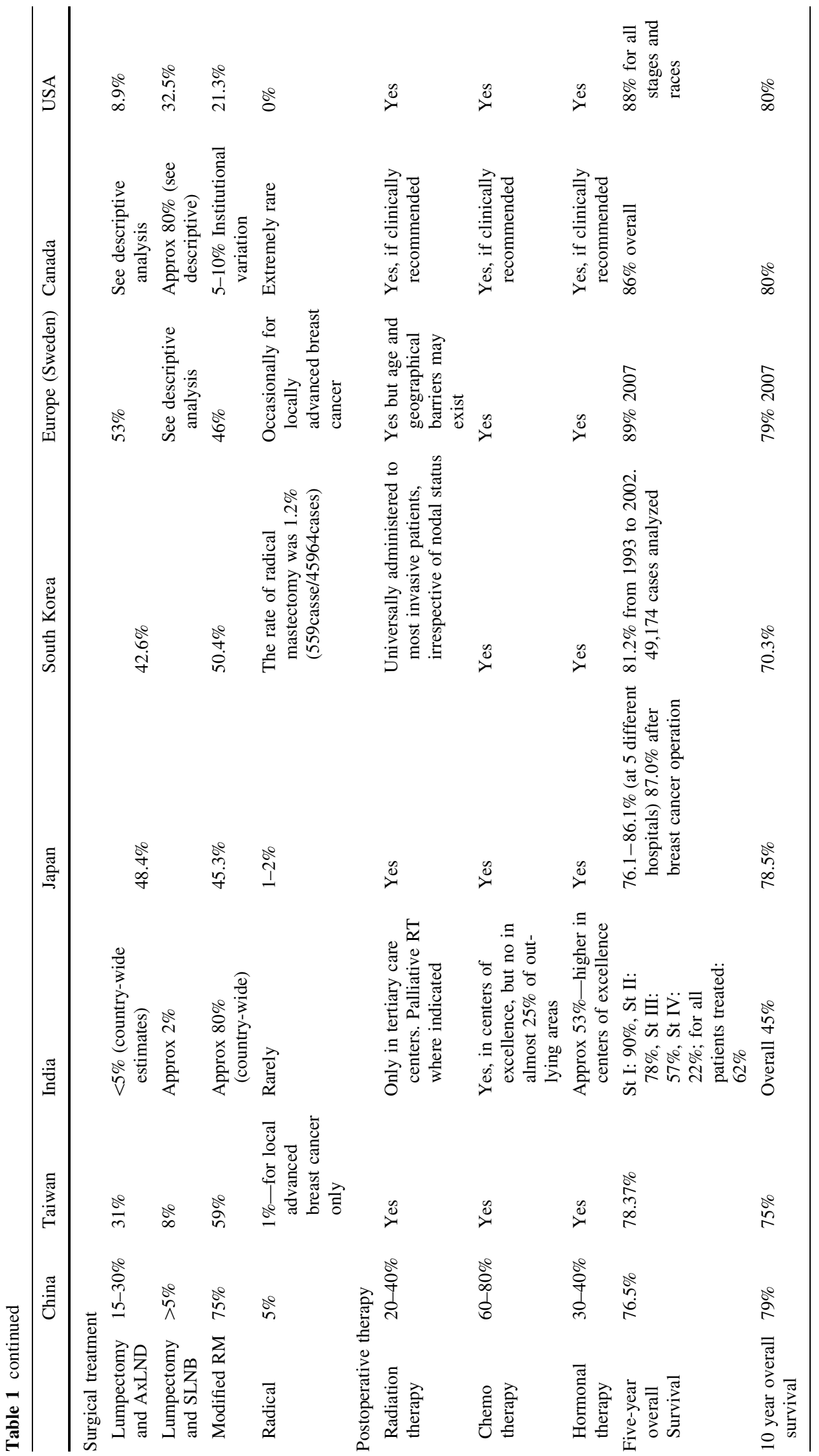

Springer 
same area. The proportion of breast-conserving surgery is $15-30 \%$ in leading hospitals and has gradually increased in recent years, according to studies published in China. But the breast-conserving surgery rate is still low in China's general hospitals. Sentinel lymph node biopsy is starting to be prevalent in urban areas, but it has not been accepted by most early-stage breast cancer patients. The prevalence of sentinel lymph node biopsy is less than $5 \%$.

Data from Fudan University Cancer Hospital indicate that an anthracycline-based chemotherapy regimen, which gradually replaced the 1990 s regimen of cytoxan, methotrexate, and 5-fluorouracil (5-FU), has become the most commonly used regimen for breast cancer in China. The taxane-containing regimen is used for node-positive disease. Herceptin is used, although not very commonly, for patients with tumors in which HER-2 is overexpressed or amplified. The indication of adjuvant chemotherapy is referred to St. Gallen 2005 consensus [6]. It is recommended that all patients with ER/PR-positive tumors receive endocrine therapy and that patients at high risk for local-regional recurrence receive postoperative radiation therapy.

\section{Survival}

In terms of survival, data from Fudan University Cancer Hospital represent the survival rates in Shanghai and some developing areas in China, but not the entire country. The Fudan experiences showed a 5-year disease-free survival rate of $76.5 \%$ and an overall survival rate of $87.6 \%$. Tenyear disease-free survival decreased to $61 \%$ and overall survival to $78.9 \%$. For patients with stage I, II, and III disease, the 5-year disease-free survival was $88 \%$ and overall survival was 94, 71 and $84 \%$, and 48 and $64 \%$, respectively.

\section{Taiwan}

\section{Data collection and incidence estimates}

In Taiwan, incidence estimates are based on data from the Bureau of Health Promotion, in the Department of Health. The incidence of breast cancer in Taiwan has increased from 6.23 per 100,000 in 1970 to 23.76 in 2000. Among all types of cancer reported in 2005, the incidence of breast cancer was the second most frequent cancer in women after age adjustment of 42.3 per 100,000 . It was the number-one killer in women with invasive cancer. The data from Taiwan's nationwide cancer registration system [7] reported 5,585 newly pathology-proven breast cancer cases in 2005. Incidence peaks in women 4050 years old and the median age was 45 . Cases in men are extremely rare, and the ratio of males to females is
$0.3: 100$. Only $2.6 \%$ of patients had first-grade relative involvement by breast cancer.

\section{Screening and methods of detection}

Several targeted screening programs have been implemented since Taiwan's Bureau of Health Promotion began nationwide breast cancer screening in 1994. The first screening strategy was carried out in 12 major teaching hospitals for the mothers, daughters, and sisters of patients with breast cancer. The screening method consisted of palpation by a breast surgeon and a mammogram and sonogram once a year for 3 consecutive years (1994-1997). A total of 83 cases of breast cancer were confirmed in 966 abnormal findings out of 4,861 families at the first visit. Another 15 cases were diagnosed in 797 abnormal findings out of 3,775 families at the second follow-up visit.

The second screening strategy (1999-2001) was carried out by public health officers for each county, who, together with volunteer breast cancer patients, recruited all female residents over 35 years of age in the county, to be examined by either a breast surgeon or a nurse at the screening location, usually the county's recreation center or a school. Women also had a cervical smear testing and a stool occult blood examination. A total of 452 breast cancer patients were diagnosed in 42,522 abnormal findings out of 852,824 females at the first visit, and 40 additional cancer cases were found in 12,078 abnormal findings out of 122,278 females at the second visit. Some of the screening teams from interested hospitals provided two additional sonograms during the screening period. The first resulted in detection of 212 breast cancer cases among 9,215 abnormal findings out of 32,102 women. The second resulted in another 40 cases diagnosed from 1,600 abnormal findings out of 4,091 women.

The third screening strategy (2004-2007) was focused on women between 40 and 49 years of age because they have the highest incidence of breast cancer in Taiwan. Because screening sonograms require clinical validation and Asian women tend to have small, dense breasts, the detection method was either a mammogram or a sonogram in the first year (Huang (2006) Randomized trial for breast cancer screening with either mammography or ultrasound among young women in Taiwan, unpublished data). The screening procedure was then alternated in subsequent years. Any abnormal finding was verified by palpation by the breast surgeon, along with other necessary diagnostic procedures. The first year of sonogram screening resulted in 20 proven breast cancer cases from 9,262 abnormal findings from 17,471 women. Mammography resulted in 26 proven breast cancer cases from 9,235 abnormal findings from 16,565 women. After three cases in each group were excluded because the patients refused surgical 
treatment, postoperative pathological examination showed ductile carcinoma in situ (DCIS) only, tumor $<1.5 \mathrm{~cm}$ and lymph node negative, tumor $>1.5 \mathrm{~cm}$ or lymph node positive by sonogram was $1 / 10 / 6$, whereas by mammogram, the finding was $10 / 5 / 8$. When this screening program was expanded to include mammograms for women between the ages of 50 and 69, 141,873 had mammograms and $15,336(10.8 \%)$ were reported as BI-RAD category 0 , 4 , or 5 . About $75 \%$ of patients with BI-RAD category 4 and 5 ( $n=11,502)$ had biopsies, and 499 were proven to have breast cancer.

\section{Treatment}

A team approach has been employed for breast cancer treatment in Taiwan. Breast surgeons, medical and radiation oncologists, social workers, and rehabilitation personnel work together under clear guidelines to obtain the best therapeutic result. The Oncology Group of the National Health Research Institute of Taiwan (a government institute under the Department of Health), published the first edition of guidelines for breast cancer management in 2001 and a second edition in 2005 [8].

The guidelines were distributed in English to all physicians and were distributed in Chinese to the public. The Bureau of Health Promotion reported in 2005 that for 5,585 newly diagnosed breast cancer cases, which were properly recorded, partial mastectomy was performed in $18.6 \%$ of all patients and $31.1 \%$ of patients with stage 0 and stage I disease. Routine axillary lymph node dissection was performed for all surgical patients.

Sentinel lymph node biopsy was validated in 1998 from a single institute, and an additional 14 hospitals have adopted this technique [9]. A nationwide questionnaire survey regarding sentinel node biopsy in 3,367 cT12N0M0 patients found that breast surgeons could obtain a false negative rate within 5\% after they accumulated experience using sentinel lymph node biopsy in 100 cases. Nevertheless, less than one-fifth of cases with negative biopsy results had omitted total axillary lymph node dissection due to medical-legal considerations.

Both preoperative and postoperative adjuvant chemotherapy are available in every hospital that follows the NCCN's Breast Cancer Therapeutic. All drugs for chemotherapy have an approval certification from the U.S. Food and Drug Administration. Every patient who underwent partial mastectomy received postoperative radiation therapy, and sometimes other areas were irradiated, according to the guidelines. Use of targeted therapy depends on the results of HER-2/neu gene testing with the DAKO method. These results appear as a routine pathological report in the majority of hospitals in Taiwan. Hormonal manipulation is a routine postoperative therapy in women whose estrogen and progesterone receptors are positive.

\section{Survival}

Bureau of Health Promotion data from 1998-2002 indicate that 5-year survival for all stages was $78.37 \%$. The 5- and 10-year survival rates are 98 and $95 \%$ for stage $0 ; 96$ and $89 \%$ for stage I; 90 and $82 \%$ for stage II; 65 and $53 \%$ for stage III; and 22 and $10 \%$ for stage IV.

India

\section{Data collection and incidence estimates}

India has no available source of comprehensive epidemiological, clinicopathological, and outcomes data for breast cancer patients. As a result, a variety of resources had to be reviewed to obtain relevant data, some of which are provided here. When no robust data source could be found, available estimates are provided. Demographic, clinical, pathological, and outcomes data on breast cancer patients managed at the Sanjay Gupta Postgraduate Institute of Medical Sciences (SGPGIMS) hospital in Lucknow, India, were collected and analyzed. We also reviewed similar data, and data on availability of breast screening, treatment facilities, and outcomes from other major cancer centers and cancer registries of India. India's national cancer registry program, under the Indian Council of Medical Research, published a 2-year (1999-2000) report of hospital-based cancer registries, and a 2-year (also 1999-2000) report of the populationbased cancer registries. These reports assessed the burden and care of cancer patients in India [10, 11]. In addition, district-wide distribution of breast cancers was derived from the All India Report 2001-2002, which was part of an atlas of cancer in India project of the National Cancer Registry Program, ICMR and is available at their web site: www.canceratlasindia.org/chapter6 [12]. We searched the published literature to obtain information on breast cancer demographics, detection, treatment, and outcomes from various Indian centers. We also searched the Internet, and other publications of cancer registries and organizations such as the World Health Organization, regional and national health agencies, and institutions and non-governmental organizations, among others. Appropriate similar publications and reports from the International Agency for Research on Cancer [13], and its GLOBOCAN database [14] were also studied. The SGPGI breast cancer database provided valid and complete information on 544 patients managed during 1998-2004 at SGPGIMS in Lucknow, India. Data from TMH, Mumbai, another dedicated breast center in India, have been incorporated so that results can be compared. 
Breast cancer accounts for about one-fourth of all cancers in Indian women and about half of all cancer-related deaths. With the exception of Chennai, all urban population-based registries in India reported breast cancer as the most common female malignancy over the years 20002001[10-12]. In earlier reports, cancer of the uterine cervix was the most common type of cancer, even in urban centers. Breast cancer remains second after cervical cancer as the most common female malignancy reported in Chennai's urban population-based cancer registry and in Barshi's rural population-based registry [12]. Data from all urban and rural population-based cancer registries in India suggest a rising incidence of breast cancer in India. Yet, the age-adjusted rates reported from various urban registries range from 24.8 to 33.4 per 100,000 [12], which is about one-third the incidence reported from Western countries such as the United States (California SF: NH White, 109.6 per 100,000) and Uruguay (Montevideo, 114.9 per 100,000) [13]. The minimum age-adjusted rates reported from the only rural population-based cancer registry in India is even lower, at a mere 7.2 per 100,000 [12], and is somewhat similar to the incidence reported from other developing countries, such as The Gambia and Jiashan, China [13].

In India, breast cancer incidence peaks among women 45-50 years of age. The average age of patients in six hospital-based cancer registries ranged from 44.2 in Dibrugarh, 46.8 in Delhi, 47 in Jaipur to 49.6 in Bangalore and Chennai [12]. Among patients at SGPGIMS in Lucknow, 26\% were younger than 35 years of age, whereas at $\mathrm{TMH}$, Mumbai, this percentage was $11 \%$ [12].

\section{Screening and methods of detection}

An organized large-scale breast cancer screening program does not exist in India. The few breast cancer screening programs available are largely targeted at small communities, covering a miniscule proportion of the Indian population, and they rely on funds derived from research grants or the screened individuals' own resources. There is no government-funded or aided mass breast screening program available; experts also advocate screening based on periodic breast examination by a physician and breast selfexamination [15]. Population-based breast cancer screening is not recommended in India due to limited resources and the lack of local statistics on mammography and breast cancers.

Nearly all Indian breast cancers are clinically detected; almost none are detected by screening. Up to two-thirds of patients present with local invasion, and 6-25\% present with metastases. Significant proportions present with T2/T3 tumors, and even more strikingly, up to one-third of all patients have skin and/or chest wall involvement
(T4a-c). Inflammatory breast cancers are seen more often in younger patients, putting them at considerably poor risk for survival. Half of the young patients managed at SGPGIMS, have stage III disease. The stage distribution of patients is worse among younger women [16].

\section{Pathologic characteristics}

In India, a large proportion of patients have high-grade tumors and hormone-receptor- negative tumors. About $60 \%$ of SGPGIMS breast cancer patients have grade 3 tumors, 66\% have auxiliary lymph node metastases, and $58 \%$ are both estrogen (ER) and progesterone (PR) receptor-negative. Data from Mumbai shows a slightly lower ER- and PR-negative rate of $47 \%$ [17]. Data on HER-2neu immuno-reactivity are scarce, but the available information hints at low rates of positive HER-2neu status: $17 \%$ of 100 breast cancer patients recently treated at SGPGIMS were found by immunohistochemistry to overexpress HER-2neu. On the whole, women younger than 35 years of age presented with larger tumors and at a later stage. They were also more likely to be ER-negative and to have a higher grade of cancer compared to the overall average.

\section{Treatment}

The standard of treatment and the infrastructure and facilities for treatment of breast cancer vary widely at various Indian hospitals. A nationally applicable standard is lacking, so the treatment available for an individual patient may vary from world-class to very ordinary or even inadequate. The first WHO-ICMR national breast cancer management guidelines meeting was held in an attempt to evolve nationally applicable treatment protocols and recommendations, taking into account the available data on breast cancer patients, and also keeping the logistics and economics in mind [18]. Only a very few patients can be considered to have received treatment at well-equipped centers that use appropriate clinical protocols. A number of compromises are made at every step of management of a patient, including the diagnostic work-up, surgical treatment, and the multi-modality therapy. For the huge Indian population of over one billion, the numbers of treatment facilities are grossly inadequate. This, along with the poor patient compliance with treatment and follow-up due to the social stigma attached to the disease, and the high cost of treatment [19], limits the effectiveness of care. Fine needle aspiration cytology is underutilized, and the preoperative diagnosis is still based predominantly on clinical and incisional or excisional biopsy. The standard of breast pathology reporting too is erratic, and may range from very 
inadequate to world class depending upon where the patient receives treatment. A significant proportion of patients undergo an incomplete or inappropriate surgical excision before they are referred to a specialist, making management challenging. At a major North Indian teaching hospital, $75 \%$ of the patients had undergone an incisional or excisional biopsy not intended for treatment of breast cancer before they were referred for management of early operable breast cancers [20]. At SGPGIMS, Lucknow, some $40 \%$ patients with early or locally advanced breast cancers are referred following an inadequate or inappropriate initial surgical procedure. Mastectomy after or without neoadjuvant chemotherapy is usually offered for all stages of breast cancers [21]. Completeness of mastectomy and that of axillary clearance if performed is questionable. Postmastectomy reconstruction is neither offered to nor accepted by most patients, as it is seen as an unnecessary burden on patient's resources.

Breast-conservation surgery (BCS) is available to patients treated at only a few selected hospitals in India, and it is performed in only about 1,000 of the estimated 100,000 breast cancer patients treated for breast cancer every year in India. Such low numbers of breast-conserving procedures are due to the relatively late stage at presentation, the few centers equipped to provide highquality breast-conserving surgery with all its components (including radiation therapy and pathology), as well as to the low acceptance rate among patients [22, 23]. At selected centers of excellence, with adequate counseling, higher proportions of cancer patients with early and postneoadjuvant chemotherapy for locally advanced disease accept breast conservation. At SGPGIMS Lucknow, and at the Breast Unit at TMH Mumbai almost $60 \%$ of early breast cancer patients are treated with BCS. Both these centers have reported that the proportion of patients undergoing BCS has been increasing, and at Mumbai, this proportion increased from $12.6 \%$ in 1997 to $59.3 \%$ in 2001 [24]. Breast-conserving surgery is also being offered to patients with locally advanced breast cancer after neoadjuvant chemotherapy, with novel innovations to make BCS practical and safer in large locally advanced breast cancer patients [23]. Sentinel lymph node biopsy is performed at very few centers, and it is estimated that only about 500 patients per year undergo this procedure.

Systemic and adjuvant treatment of breast cancer varies in availability, use, and standards between various centers in India, with a wide variety of chemotherapy regimens in use. Although most physicians agree that anthracyclinebased chemotherapy combinations are an appropriate firstline approach to most patients, the cytoxan, methotrexate, and 5-FU (CMF) combination is still used in a large proportion as first-line therapy because of its low cost. At a community hospital in South India, of 122 breast cancer patients treated, 5-FU, Adriamycin, cytoxan (FAC) regimen was used in $51.2 \%$ and CMF in $48.8 \%$ patients [21]. Taxanes are used in a few patients, with their use limited by their high costs and toxicity. Tamoxifen is the most widely used endocrine therapy, and the proportion of postmenopausal patients treated with aromatase inhibitors, though increasing, is very small. Surgical oophorectomy is still a widely practiced and valid choice in the vast majority of hormone responsive premenopausal and advanced stage breast cancer patients. Radiation ablation and goserlin are relatively less effective and expensive. Targeted therapies like trastuzumab are out of reach for most Indian patients. There are very few radiation therapy units, and as a result only about $20 \%$ of patients are treated with postmastectomy radiation. The lack of adequate numbers of radiation therapy facilities is a major hurdle in setting up more breast-conservation therapy programs. Compliance with adjuvant treatment among Indian patients outside major institutions is rather poor due to the high cost, extended treatment duration, and toxicity $[25,26]$.

\section{Survival and mortality estimates}

Although the incidence of breast cancer in India is lower than that in Western countries, the mortality rates are disproportionately higher [25, 26]. Thus breast cancer remains the single largest cause for cancer deaths in Indian women, similar to the rest of the world. Globally, it accounts for about $15 \%$ of all cancer deaths in women, killing some 471.2 women per 100,000 annually [18]. The 5-year survival rate of breast cancer patients reported at Bangalore's population-based cancer registry was 42.3\% [27]. The Madras Metropolitan Tumor Registry reported survival rates of 80,58 , and $48 \%$ at 1 year, 3 years, and 5 years, respectively [28]. The 5-year overall survival rate for patients treated and followed at SGPGIMS Lucknow is $62 \%$, with the 5-year survival rate of $90 \%$ for stage I, $78 \%$ for stage II, $57 \%$ for stage III, and $22 \%$ for stage IV patients. The overall 10-year estimated survival of SGPGIMS Lucknow patients was $35 \%$; with a 10-year survival rate of $75 \%$ for stage I, $55 \%$ for stage II, $35 \%$ for stage III, and $5 \%$ for stage IV patients $[25,26]$.

Japan

\section{Data collection and incidence estimates}

There is no nationwide cancer registry in Japan. All clinical data has been collected as cases registered by the Japanese Breast Cancer Society. In 2004, 14,749 cases were registered. Survival data has been collected since 1992, with a 
total of 2,320 cases as of 1992. (http://www.jbcs.gr.jp/ english/english.html). In 2005, the number of new cases per year was estimated to be 41,490 . The percentage of the entire population of Japanese women estimated to have breast cancer was $0.06 \%$. Since 1998 , breast cancer is the most common cancer among women with an age-adjusted rate of 43.6 in 1998. Breast cancer incidence peaks in women between 45 and 49 years of age. The ratio of breast cancer incidence in Japan to the U.S. is 1:3. The incidence of male breast cancer is estimated at $0.4 \%$, based on 2,320 cases registered in Japan Cancer Society in 1992.

\section{Stage of initial presentation}

According to the Japan Breast Cancer Society, among 14,166 registered cases, $43.6 \%$ presented with stage II disease.

\section{Screening and methods of detection}

Screening mammography has been used. For women over 40 , it was recommended by the Japanese Breast Cancer Society that they have a mammography once every 2 years. Self examination has also been recommended. A healthcare taker examination was not available.

\section{Treatment}

According to statistics from the Japanese Breast Cancer Society in 2003, 48.4\% of patients had lumpectomy and sentinel lymph node biopsy or axillary lymph node dissection, $45.3 \%$ had modified radical mastectomy, and $1-2 \%$ had extended radical mastectomy. Postoperative therapy includes radiation, chemotherapy, and hormonal therapy for all patients as needed.

\section{Survival and mortality estimates}

The overall 5-year survival rate is $82.2 \%$ and ranges from 73.1 to $86.1 \%$ among the 14 cancer hospitals contributing data. The crude breast cancer survival rate based on this data correlates with tumor size. With $5.1 \mathrm{~cm}$ at approximately $50 \%$, and $0.5-2 \mathrm{~cm}$ the crude 5-year survival rate is about $90 \%$ and the disease-free 10-year survival is $65-77 \%$. Although breast cancer deaths in the U.S. have decreased in the past 40 years, according to data from the U.S. SEER program in 2001, deaths from breast cancer continue to increase in Japan, according to the Japanese Government's Health and Welfare statistics in 2003. In 2005, total deaths due to breast cancer were estimated to be 10,808 . The death rate was 10.8 per 100,000 populations, a 6.46-fold increase from 1950 to 2000 [29].

\section{South Korea}

\section{Data collection and incidence estimates}

The incidence of breast cancer in South Korea has continuously increased in recent years. Breast cancer has emerged as the most frequent malignancy among Korean women since 2001. The Ministry of Health and Welfare of the Republic of Korea started the Korea Cancer Center Registry (http://www.kbcs.or.kr) in 1980 and established a National Cancer Incidence Database [30].

The Korean Breast Cancer Society (KBCS) has been conducting a nationwide survey on breast cancer and its clinical and epidemiological characteristics since 1996, in the form of recorded cancer registration sheets. In 2001, an on-line registration program was started, and 39 medical schools (71 hospitals), 27 general hospitals, and 7 private clinics now participate in this program. To date, 46,667 cases from the on-line registration program were analyzed and reported in 2006 [30]. The collection and management of survival data was conducted with the Korean Breast Cancer Society (KBCS). Survival was analyzed from a total of 49,174 cases from 1993 to 2003.

Breast cancer is the fifth leading primary cancer in South Korea, but has been the most frequent malignancy among Korean women since 2001. Since 1996, the incidence of breast cancer has increased steadily, from 3,801 cases in 1996 to 9,668 newly diagnosed cases in 2004. The accrued incidence rate of breast cancer in 2004 was 40.5 per 100,000 with an increase since 1996 of 16.7 per 100,000. In Korea, breast cancer incidence peaks between the ages of 40 and 49 years (median: 47 years). In 2004, 60\% of women diagnosed with breast cancer were pre-menopausal.

\section{AJCC stage of initial presentation}

Most of the women_about $60 \%$ - presented with a painless lump. Asymptomatic breast cancer was detected on screening $18 \%$ of the time. The diagnosis was made by fine needle aspiration $27 \%$ of the time. Invasive carcinoma incidence was $87 \%$ and that of ductal carcinoma in situ (DCIS) was $8.5 \%$. According to the American Joint Cancer Commission (AJCC) staging system, breast cancer in South Korea is diagnosed as stage II in about $40 \%$ of cases. Approximately $5.1 \%$ of patients are diagnosed as stage IIIC. The proportion of early cancers diagnosed as stage I has increased strikingly, from $19.6 \%$ in 1996 to $36 \%$ in 2004 .

\section{Treatment}

Modified radical mastectomy was performed most frequently in the 1990s, but its use has decreased from $79.7 \%$ in 1996 to $54 \%$ in 2006 . The use of breast-conserving therapy 
has been rapidly increasing, from $18.7 \%$ in 1996 to $42.6 \%$ in 2006. Sentinel lymph node biopsy has been used with increasing frequency. The rate of reconstruction after mastectomy is $8.5 \%$.

\section{Survival}

From 1993 to 2002, a total of 49,417 cases were analyzed, 46,355 of which were invasive cancer and 2,819 of which were noninvasive [31, 32].

The 5-year survival rate for invasive breast cancer was $80.3 \%$; 10-year survival was $70.3 \%$. For DCIS, the 5-year survival rate was $99 \%$. The increase in survival may be due to the increase of early breast cancer detection and better treatments. The 5-year survival rate for Korea is similar to that of Japan but lower than that of United States.

\section{Sweden}

\section{Data collection and incidence estimates}

Data on patients treated for breast cancer are gathered through the oncologic centers in six geographical regions and reported to the Swedish Cancer Register. Details regarding diagnosis, actual treatment details, and pathology and adjuvant treatment are available for each region. Sweden's National Board of Health and Welfare is responsible for official statistics [33]. Breast cancer in Sweden accounts for $30 \%$ of all female cancers, and $0.2 \%$ of all male cancers. The annual increase in breast cancer is $1.5 \%$ over a 20 -year period and 1.6 for a 10 -year period.

\section{Screening and methods of detection}

In Sweden, screening is offered to women between the ages of 40 and 74 where the regional county makes decisions on age groups 40-49 and 70-74. Screening compliance is approximately $65-80 \%$, and is lower in urban areas and higher in rural areas. Self-examination or exams performed by healthcare workers is not recommended.

\section{AJCC stage of initial presentation}

In the Stockholm Gotland region in 2006, 1,524 new cases of breast cancer were registered. There were 1,385 cases of invasive carcinomas and 139 non invasive. Fifty-six percent of the invasive cancers were stage I, $37 \%$ stage II, $5 \%$ stage III, and $2 \%$ stage IV.

\section{Treatment}

Treatment protocols presented in national guidelines form the basis for treatment for the Swedish population. Treatment outside protocols is rare and almost exclusively performed within randomized controlled trials. Social security covers costs for diagnostic work-up and treatment. The woman is charged a minimal fee for service for screening but not for diagnostic work-up. A fee exists also for medical visits up to a certain sum, which is similar for prescribed medication. No patient is declined treatment for economic reasons.

The regional breast cancer database in Stockholm-Gotland, has gathered information since 1977, when the first treatment protocol was implemented [34]. In 2004, 1,305 women had surgery as a primary treatment modality. The mastectomy versus breast conservation rate was 41 vs. $58 \%$. In 1997, sentinel lymph node biopsy was introduced in Sweden, and all such procedures were concurrently registered. After the completion of national feasibility and validation studies sentinel lymph node biopsy was then performed in selected cases (unifocal, clinically nodenegative patients with $\mathrm{T} 2<3 \mathrm{~cm}$ ). Stockholm data from 2005 showed that sentinel node biopsy was performed in $44 \%(610 / 1,392)$ of all breast surgery procedures for invasive and noninvasive cancer [35, 36]. Since 2008 a web-based national breast cancer registry collects data on detection, treatment, tumor-specific data, complications, lead times, and planned treatment. The registry relates to quality goals set by the profession and the National Board of Health and Welfare. Sentinel node biopsy was the final axillary procedure in $57 \%$ of all operated breast cancers in Sweden in 2008 (www.karolinska.se/oc).

\section{Survival}

The current overall survival rates on a national level are $86 \%$ at 5 years and $75 \%$ at 10 years (http://www. socialstyrelsen.se/Publicer).

\section{Canada}

\section{Data collection and incidence estimates}

Breast cancer is an important public health issue in Canada where considerable resources are expended annually for prevention and diagnosis at an early stage.

Annually the National Cancer Institute of Canada, the Canadian Cancer Society, the Public Health Agency of Canada, provincial cancer registries, and Statistics Canada, accrue, analyze and publish the "Canadian Cancer Statistics." The main purpose of these published data is to provide health professionals, researchers, and policy-makers information on common cancers in Canada with respect to incidence, mortality, cancer distribution, and survival. Data provided for 2007 are estimates and not actual data. These estimates are calculated projections based on past numbers and trends [37]. 
The most common cancer in Canadian females is breast cancer, with an estimated 22,300 cases this year. There will be an estimated 170 cases in males. Breast cancer continues to be the leading cause of cancer among Canadian women at $28.9 \%$ of estimated new cases. However, lung cancer in 2007 will continue as the leading cause of cancer death in Canadian women at $26 \%$ of cancer-related deaths, followed by breast at $15.5 \%$. In 2003 subgroup analysis by adult age group $(20-49 ; 50-69 ; 70+)$ showed that breast cancer was the most common female cancer in all age groups. However, breast cancer was the leading cause of death in young women (20-49) and ranked second in the 50-69 age group, following lung cancer. Between 1978 and 1999 the incidence of breast cancer rose steadily but has now stabilized. This increase was probably due to an increase in mammographic screening and improved quality of screening during the 1980s and 1990s. The age-standardized incidence rate per 100,000 rose from 86.1 in 1978 to 104 in 2007. Other reasons contributing to the increase in incidence are likely modifiable risk factors such as increased use of hormone replacement therapy, higher prevalence of obesity, delayed first pregnancy or null parity, and greater consumption of alcohol. Age-standardized mortality rates have been declining since the mid 1980s, and since 1999 this trend has accelerated to $1.2 \%$ per year. Between 1978 and 2007 the age-standardized mortality rates per 100,000 went from 29.5 to 22.9 [38].

Earlier detection and increased use of adjuvant hormones and chemotherapies have contributed to this decline. Separating the impact of screening and treatment is challenging. In a U.S. study by Berry et al., the input of each contributed about equally to improved breast cancer outcomes [39].

Overall in Canada, the lifetime risk of developing breast cancer as a woman is $11 \%$ ( 1 out of 9 women), and the lifetime probability of dying from cancer is $3.7 \%$ ( 1 in 27 women) [38].

\section{Survival}

Long-term trends in survival from Ontario between 1975 and 1999 showed consistent improvement in survival; however, it increased significantly after 1989 at $0.8 \%$ per year. The trend toward improved survival was strongest in the age groups most affected by screening (ages 50-69) [38]. Five-year relative survival ratios by stage of disease are stage I $96 \%$, stage II $86 \%$, stage III $59 \%$, and stage IV $26 \%$, as recorded between 1994 and 1997 at a Canadian regional cancer center [40]. The Canadian 5-year relative survival rate for breast cancers diagnosed from 1995-1997 is $86 \%$ overall [37].

Analysis of hospital stays for breast cancer patients between 1981 and 2000 by Neutel et al. demonstrated that between 1997 and 2000 approximately 65\% of cases presented locally, followed by regional presentation at 22 and $12 \%$ with distant metastases. In 1981-1984 this compared with local presentation at $58 \%$, regional at $14 \%$, and distant presentation at $28 \%$ [41].

\section{Treatment}

In the last two decades a dramatic shift in surgical practice has brought breast-conserving surgery to the forefront. The declining use of mastectomy for breast cancer in Canada has been likely linked to the publication of NSABP B-06 in March 1985, and earlier declines were probably influenced by the Italian trials and gradual acceptance through discussion and practice of clinical trials in North America. Further, in 1991, the National Institutes of Health in the U.S. published its consensus conference recommendations and its support for breast-conserving surgery in early stage breast cancers. In 1998 when the Canadian guidelines were first published, the recommendations were similar to the NIH recommendations for mastectomy and breast conservation. In Canada, mastectomy rates decreased from 62.2 to 37.9 (per 100,000) between 1981 and 2000. The largest declines occurred between 1984 and 1985. In contrast, breast-conserving surgery rates mirrored the numbers for mastectomy. They rose rapidly until 1986, and between 1981 and 2000, breast-conserving surgery increased from 20.4 to 38.2 . In all designated time periods analyzed between 1981 and 2000 Quebec had the lowest provincial mastectomy rates and the highest breast-conserving surgery rates. The Atlantic Provinces showed an opposite pattern, whereas intermediate surgical practice patterns were observed in the west and in Ontario [42]. Shen et al. found that in the mid-1990s, $80 \%$ of cases were treated by mastectomy and $20 \%$ by breast-conserving surgery [43].

As surgical patterns continue to evolve, sentinel node biopsy (SLN) has also moved to the forefront. Practice guidelines do exist in Canada, and these are rooted in the criteria proposed by the American Society of Breast Surgeons and the American College of Surgeons Oncology group. The great majority of cases are performed in a university practice setting and by surgeons with surgical oncology training. Rates at individual institutions vary greatly depending on staff training, university affiliation, and geographical location [44].

In the years to come we should see research showing trends of SLN practices and how this compares with axillary node dissection as we have seen for mastectomy and breast-conserving surgery rates. For now in Canada most of what we have in the literature are survey data, which are not a reliable reflection of practices. Results of randomized trials will further guide our practice of SLN biopsy. 


\section{Screening}

Every province and territory has an organized screening program inviting women for a biennial mammogram and annual clinical breast exam for women 50-69 years of age. None of the programs have achieved the nationally established target of $70 \%$ participation; however, most provinces have shown an increasing trend in participation of these organized programs. This is most likely due to recruitment campaigns and a greater emphasis on accessibility to screening. In Canada about $61 \%$ of women 50-69 years of age reported having a screening mammogram in 2003, which is a considerable increase from $53.5 \%$ in 2000/01. Also, in some provinces, greater numbers of women report having a screening mammogram than is the numbers reported by the corresponding provincial program. This is likely because screening mammography is also available through centers not affiliated with organized programs [37].

Both before and after menopause breast cancer is the most common cancer in Canadian females. Incidence remains high and stable, mortality is declining significantly at all ages, and survival is increasing.

\section{United States}

\section{Data collection and incidence estimates}

Data on breast cancer incidence, the female/male ratio of 101:1, and 5-year survival in the U.S. are taken from the journal CA [45]. The median age of diagnosis comes from the National Cancer Institute's Surveillance, Epidemiology, and End Results (SEER) database [46].

All of the data on frequency of mastectomy and lumpectomy come from the Memorial Sloan-Kettering Cancer Center (MSKCC) Combined Database (comprising all patients undergoing surgery for breast cancer at the MSKCC), and the data on method of detection come from the NCCN patient-reported survey given to all new patients seen at the MSKCC for a breast cancer diagnosis. Some caveats apply to interpreting these data. First, the data do not distinguish between patients with DCIS and invasive cancers; treatment is quite different in each group. Second, the final operation is the operation of record; a significant proportion of patients (perhaps 20-30\%) in whom breast conservation was attempted eventually had mastectomy for persistently positive margins or at their own request. Finally, the data do not examine the extent to which age, family history, personal history of cancer, and genetic predisposition (especially germline BRCA mutations) affected the choice of treatment.

Recent cancer statistical publications in the United States report that in women, breast cancer has the highest incidence of any cancer, at $26 \%$. The lowest incidence is in New Mexico (115 per 100,00 per year) and the highest is in Washington (147 per 100,000) [45]. The increased incidence of breast cancer reflects an increased proportion of ductal carcinoma [45]. Breast cancer incidence varies among ethnic groups, with "Non-Hispanic White" having the highest incidence, and Koreans, the lowest [47].

\section{AJCC stage of initial presentation}

There is significant geographic variability in the initial patterns of breast cancer presentation in the United States [48]. From 1999 to 2003, a total of 811,652 breast cancer patients were reported to the National Cancer Data Base of the American College of Surgeons Commission on Cancer. However, only 777,884 had complete stage and age information recorded. The greatest percentage of stage I was reported by the Northeast region at $61 \%$, whereas the lowest percentage was recorded in the South at $57 \%$. Conversely, stages III and IV disease were reported higher in the South when compared with the rest of the United States (11.2 vs. 10.3\%) [48].

\section{Screening and methods of detection}

Mammography screening increased among all age groups of U.S. women significantly between 1987 and 1992. Differences in mammography screening between black women and white women disappeared by 1992 . However, reported screening among Hispanic women was still lower that that of non-Hispanics. Higher education, more income, and urban environment are associated with increased reported mammography screening [49].

\section{Treatment}

Radiation therapy, chemotherapy, and hormonal therapy are available to all our patients. We do not have any current institution-based 10-year survival data; a single-surgeon series comprised of patients treated between 1965 and 1978 at MSKCC was published in 1991 [50]. For early breast cancer, sentinel lymph node biopsy has been adopted as a standard approach [51-53].

\section{Survival and mortality estimates}

In the United States, cancer survival has been shown to be correlated with income and socioeconomic status [54]. Socioeconomic status has been linked to decreased use of optimal breast cancer care in the areas of screening, access to treatment, access to clinical trials, co-morbidity, delivery of care, treatment recommendations, and healthcare workforce disparities. According to a recent Medscape 
CME presentation by Newman L. E. (http://cme.medscape. com/viewprogram/17141), disparities in breast cancer treatment and outcomes may be related to ethnic background, socioeconomic resources, delivery of care, tumor biology, genetics, lifestyle, reproductive experiences, environmental exposure, and diet and nutrition factors.

Breast cancer ranks second to lung cancer as the cause of death among women in the United States [55]. The mortality rate for breast cancer has decreased, however, from $33 / 10,000$ per year in 1991 to $25 / 100,000$ per year in 2003, suggesting that breast cancer is being diagnosed early and can be cured.

\section{Discussion}

Several major international congresses have been held optimizing breast cancer care among women from several countries, and all of them have noted East-West differences [56]. The goal of the Breast Surgery International symposium attended by surgeons from many nations during the 2007 Montreal International Surgical Week was to discern whether breast cancer characteristics other than incidence differ between Asian and Western countries. The summaries presented by breast cancer experts from China, Taiwan, India, Japan, South Korea, Sweden, Canada, and the Unite States confirm previous studies in showing a striking difference in peak incidence, which is between 40 and 50 in Asian countries, and between 60 and 70 in Western countries. The relative contribution of biological, genetic, and environmental factors to this difference clearly merits further study if we are to determine whether breast cancer is the same disease in Asia as in the West. Incidence is increasing in Asia and in the West, but mortality is rising in Asian countries and decreasing in the West.

Female breast cancer in the Western world has shown a steady yearly increase of $1.5 \%$. The most commonly cited reasons for this increase are breast cancer awareness, early detection by screening programs, and reliable diagnostic tools. Screening on a national basis exists in several countries, namely Canada, the United Kingdom, the Netherlands, Finland, Sweden, Norway, Australia, and Singapore. Preventive measures, and effective surgical, endocrine, cytotoxic, and radiation therapy account for the excellent survival data and the slight decline in mortality during the last decade in the West. Although these countries do not represent the world as a whole, the data collected give us a glimpse of the breast cancer statistics in these countries and are a critical first step toward broader global representation in the future.

The importance of a global approach to breast cancer is to share the preventive measures and appropriate treatments from more advanced countries rapidly with developing countries [1, 3, 56]. Although the infrastructure for reporting cancer varies widely in the countries that participated in the symposium, and the data often are not representative of the entire country, important global comparisons can still be made. The trend shown in this report is that the incidence of breast cancer increases as countries become more urbanized. The traditional approach of mastectomy gives way to more lumpectomies. Although sentinel lymph node biopsy is a standard staging procedure [51-53] for early breast cancer in the U.S., and perhaps in Canada and Sweden, it is still a new technique and being evaluated in Asia.

To attempt to answer the question posed by the title of this article-is breast cancer the same disease in Asia as in Western countries? - we can first take an overview of the data presented at the ISW 2007 meeting. The differences in the data obtained are striking. If we put to one side data on the stage at presentation of cancer (which is most likely related to access to service, and will be a major determinant of survival) and look instead at incidence rates, it is clear that incidence rates for breast cancer are uniformly lower in Asia than they are in the West.

National cancer registry data have confirmed that breast cancer incidence rates differ between Asia and the West in two major ways: first, the overall rates are far lower [57, 58]; second, the shape of the age-incidence curve is markedly different and importantly, this difference mirrors differences in the age-incidence distribution for $\mathrm{ER}+$ and ER - breast cancers [59]. When breast cancer incidence is plotted as a log-log plot, the linear increase in breast cancer is inflected around the menopause ("Clemmesen's hook") [60]. When one plots ER+ and ER- breast cancers separately, the slopes are quite distinct, with the ER+ risk continuing after the menopause, whereas the ER- clearly diminishes [61]. This is also seen when breast cancer histological subtypes are plotted independently; for example, medullary breast cancers (which are very frequently ER-), show a sharp falling-off in incidence after the menopause [62]. There is also evidence for this bimodality in risk profile when intrinsic breast cancer subtypes [63], rather than ER status, are used to subdivided breast cancers. The median age at diagnosis for luminal A and B tumors was 74 years, whereas it was 52 years for basal and HER2+ subtypes [62], suggesting that bimodality is an essential feature of breast cancer worldwide, and that the two main differences between breast cancer in the West and in Asia is the prevalence of the relevant risk factors and the access to health care.

It is possible that some of the difference in risk profiles between Western and Asian women is related to the structure and gene expression profile of the normal breast. For example, normal breast epithelium is much more likely 
to be $\mathrm{ER}+$ in Western women than in Japanese women [64]. By contrast, the data on breast density is conflicting, and in general, Asian women in Asia, compared with whites in North America, seem to have low breast cancer rates despite having small, dense breasts, although it may be that within Asian populations, risk is proportional to density [65]. The relationship between density and risk is also uncertain when studying Asians born and/or living in North America, as the data are conflicting [66-68], and it is not clear if correcting for hormonal factors and body mass index can completely explain any differences observed.

Other more general factors, such as total energy balance, are likely to be important as well, and may interact with breast-specific effects as well as nonspecific hormonal "drivers," to modulate the risk for breast cancer. This leads on to consider what happens when environments change but genes stay the same.

When families move from Asia to the West, they take their genes with them, but to a large extent, they leave their environment behind. While, naturally, some aspects of home life will be similar for new immigrants, they will inevitably be exposed to the new world they find themselves in. Data clearly show that when the female offspring of Asia-born couples are brought up in North America, their risk for breast cancer is substantially increased compared with the rates observed in Asia-born Asian females [69]. Nevertheless, incidence rates among Asia-born Asian females are clearly increasing at a rapid pace [57, 58, 70, 71]. The only plausible explanation for these findings is that some aspect of what is variously called urbanization, "modern living," and "Westernization" is affecting Asian breast cancer rates quite rapidly and probably in an irreversible way. This process seems to be affecting different populations in slightly different ways.

The second way to study this phenomenon is to study a population in which the environment is roughly the same but the genes are different. Singapore is a city, an island, and a state and is composed of three main ethnic groups: Chinese, Malays, and Indians. In the past 40 years, Singapore has experienced a rapid economic growth. From 1968 to 2002 , $85 \%$ of 16,178 female breast cancers in Singapore occurred in Chinese, $10 \%$ in Malays, and $5 \%$ in Indians. Over this time period, breast cancer has increased year by year; by $3.1,2.8$, and $1.7 \%$ per year for Chinese, Malays, and Indians, respectively. Despite the rather similar external environments, current breast cancer rates are somewhat different in the three ethnic groups, and they have changed in different ways over the time period studied. Essentially, what is seen is that up to age 50, rates are lowest in Indians, but rise in a similar fashion in the other two groups. After age 50, the pattern is different; the rates for Indians continue to rise to age 70 years, whereas for Chinese, and even more so for Malays, the rates decrease. Birth cohort effects are also quite marked, especially in the Malays, where women born in 1971-1975 have seven times the risk of breast cancer as those born in 1921-1925 [71]. Interestingly, all three populations experienced very similar and dramatic declines in fertility, but the temporal trends in breast cancer incidence differ between the groups, suggesting that other factors are playing a role in determining breast cancer risk.

As discussed above, one can specifically analyze subtypes of breast cancer. Ideally, one would like to be able to study many different breast cancer subtypes at the same time in different ethnic and geographical subgroups (e.g., basal-like, luminal-like, etc.) but this is impractical at present. It is possible, however, to study estrogen receptor-positive $(\mathrm{ER}+)$ and estrogen receptor negative $(\mathrm{ER}-)$ subtypes, as a large proportion of laboratories capable of making a diagnosis of breast cancer will be able to offer basic immunohistochemical analysis for the presence or absence of the estrogen receptor. When one compares ER+ and ER - breast cancer separately, some very interesting findings emerge. First, when considering Western countries, it is clear that ER+ and ER - breast cancers are different diseases [61].

The proportion of ER+ to ER- breast cancers does seem to differ somewhat by North American ethnic groups, with blacks having the highest percentage of breast cancers being of the ER-/PR - phenotype (23\%), followed by Hispanics (18.1\%) and Filipinos (17.4), with Japanese and whites having almost identical percentages (14.1 and $14.7 \%$, respectively) Therefore, other than blacks compared to all others, variations in ER status by ethnicity do not seem to be large [72]. Interestingly, the age peak for ER-/PR - tumors occurs much later in life for Japanese Americans and native Hawaiians (64-65 years) than for whites (50 years) [72]. Detailed data are generally not available for Asian countries.

One might expect that worldwide differences in incidence rates would be greater for ER+ cancers than they are for ER - cancers, but it is difficult to prove this as women in developing countries will have less access to laboratory and imaging technologies, and this could lead to various biases that would diminish the number of ER+ breast cancers diagnosed [73]. Nevertheless, a detailed analysis of the literature has revealed significant differences in the effect of known epidemiological risk factors (such as parity, age at first birth, and body mass index) on ER+ compared with ER- breast cancer [74, 75]. In general, ER - cancers seem somewhat insensitive to most of the traditional risk factors associated with breast cancer, making prediction and prevention of such cancers rather difficult. Conversely, since ER+ cancers are sensitive to "lifestyle" factors, one would predict that is it ER+ cancers that are going to increase rapidly in numbers in Asians as they become more Westernized [76]. 
Taken together, these findings suggest the following seven conclusions:

- Rates of breast cancer are uniformly many times lower in Asia than in the West

- The peak age of onset in Asia is 45-50 years of age, whereas it is 55-60 years in the West

- There is a clear deficiency of post-menopausal ER+ breast cancers in Asians living in Asia compared with Western populations

- The decrease in age-specific breast cancer rates with age seen in Asian women cannot be completely explained by a deficiency of $\mathrm{ER}+/ \mathrm{PR}+$ cases

- Outside of some special situations, there is no biological difference in the breast cancers occurring in Asia and the West

- The observed differences are most likely mainly attributable to different risk factors acting differentially on two (or more) different types of breast cancer

- As Asia becomes urbanized and Westernized, we can expect significant changes in the currently observed patterns, towards Western-type data.

In response to these conclusions, the participants in the Breast Surgery International symposium made a recommendation to establish an international prospective database, which could perhaps be funded by the World Health Organization. Such a database would be a more reliable way to determine similarities and differences in breast cancer among women from diverse countries and different ethnic and genetic backgrounds, as the data will be prospectively collected.

Acknowledgment We appreciate the editorial assistance of Sue Louiseau, California Pacific Medical Center Research Institute, San Francisco, CA, USA.

Open Access This article is distributed under the terms of the Creative Commons Attribution Noncommercial License which permits any noncommercial use, distribution, and reproduction in any medium, provided the original author(s) and source are credited.

\section{References}

1. Anderson BO, Jakesz R (2008) Breast cancer issues in developing countries: an overview of the breast health global initiative. World J Surg 32:2579-2585

2. Hortobagyi GN, de la Garza Salazar J, Pritchard K et al (2005) The global breast cancer burden: variations in epidemiology and survival. Clin Breast Cancer 6:391-401

3. Porter P (2008) "Westernizing" women's risks? Breast cancer in lower-income countries. N Engl J Med 358:213-216

4. Green M, Raina V (2008) Epidemiology, screening and diagnosis of breast cancer in the Asia-Pacific region: current perspectives and important considerations. Asia Pacific J Clin Oncol 4(Suppl 3):S5-S13
5. Parkin DW, Bray F, Ferlay J (2005) Global cancer statistics 2001. CA-Cancer J Clin 55:74-108

6. Senn H, Thürlimann B (2005) The international breast cancer treatment consensus St. Gallen-2005: departure to new risk selection and new treatment options. Breast 14:427-428

7. Taiwan Public Health report (2005) Annual report of bureau of health promotion. Department of Health, Executive Yuan, Taiwan, ROC

8. Liu TJ et al (2005) Guideline of breast cancer management. National health research institute, Oncology Group, Taiwan, ROC

9. Liu TJ (2007) Validation of sentinel lymph node in Taiwan breast cancer patients. J Formosa Med Assoc 106:126-134

10. National Cancer Registry Program Indian Council of Medical Research (2005) Two year report of the population based cancer registries 1999-2000. Incidence and distribution of cancer, Bangalore, India

11. National Cancer Registry Program Indian Council of Medical Research (2005) Two year report of the hospital based cancer registries 1999-2000. An assessment of the burden and care of cancer patients, Bangalore, India

12. Nandakumar A, Gupta PC, Gangadharan P, Visweswara RN (eds) (2004) Summary of specific sites: breast (ICD-10:C50)-females. In: Development of an atlas of cancer in India: first all India report: 2001-2002, Bangalore, India

13. Parkin DM, Whelan SL, Ferlay J et al (eds) (2002) Cancer incidence in five continents, volume VIII, IARC scientific publications no 155. Lyon, France

14. IARC (2002) Breast cancer incidence in different regions GLOBOCAN 2002. IARC. http://www-dep.iarc.fr/GLOBOCAN

15. Mittra I (1994) Breast screening: the case for physical examination without mammography. Lancet 343:342-344

16. Mathew A, Pandey M, Rajan B (2002) Do younger women with non-metastatic and non-inflammatory breast carcinoma have poor prognosis? World J Surg Oncol 2:2

17. Chopra R (2001) The Indian scene. J Clin Oncol 19:106-111

18. Tata Memorial Hospital Breast Cancer Working Group. The first WHO guidelines development meeting for breast cancer. http:// www.whoindia.org/LinkFiles/Cancer_tmh_breast.pdf. Accessed 26 Oct 2009

19. Agarwal G, Pradeep PV, Aggarwal V et al (2007) Spectrum of breast cancer in Asian women. World J Surg 31:1031-1040

20. Tewari M, Pradhan S, Kumar M et al (2006) Effect of prevailing local treatment options of breast cancer on survival outside controlled clinical trials: experience of a specialist breast unit in North India. World J Surg 30:1794-1801

21. Kuraparthy S, Reddy KM, Yadagiri LA et al (2007) Epidemiology and patterns of care for invasive breast carcinoma at a community hospital in Southern India. World J Surg Oncol 5:56

22. Raina V, Bhutani M, Bedi R et al (2005) Clinical features and prognostic factors of early breast cancer at a major cancer center in North India. Indian J Cancer 42:40-45

23. Aggarwal V, Agarwal G, Lal P et al (2008) Feasibility study of safe breast conservation in large and locally advanced cancers with use of radiopaque markers to mark pre-neoadjuvant chemotherapy tumor margins. World J Surg 32:2562-2569

24. Dinshaw KA, Budrukkar AN, Chinoy RF et al (2005) Profile of prognostic factors in 1022 Indian women with early-stage breast cancer treated with breast-conserving therapy. Int J Radiat Oncol Biol Phys 63:1132-1141

25. Agarwal G, Ramakant P, Sánchez Forgach ER et al (2009) Breast cancer care in developing countries. World J Surg 33:2069-2076

26. Agarwal G, Ramakant $P$ (2008) Breast cancer care in India: current situation and challenges for the future. Breast Care 3:21-27

27. Murthy NS, Agarwal UK, Chaudhry K et al (2007) A study on time trends in incidence of breast cancer-Indian scenario. Eur J Cancer Care 16:185-186 
28. Gajalakshmi CK, Shanta V, Swaminathan R et al (1997) A population-based survival study on female breast cancer in Madras, India. Br J Cancer 75:771-775

29. Shibuya K, Mathers CD, Boschi-Pinto C et al (2002) Global, regional estimates of cancer mortality, incidence by site: II. Results for the global burden of disease 2000. BMC Cancer 2:37

30. The Korean Breast Cancer Society (2006) Nationwide korean breast cancer data of 2004 using breast cancer registration program. J Breast Cancer 9:151-161

31. The Korean Breast Cancer Society (2002) Clinical characteristics of breast cancer patients in Korea in year 2002. J Korean Breast Cancer Soc 5:217-224

32. The Korean Breast Cancer Society (2004) Nationwide Korean breast cancer data of 2002. J Korean Breast Cancer Soc 7:72-83

33. Halmin M et al (2008) Sweden national health care system. Acta Oncol 47:216

34. http://www.karolinska.se/Verksamheternas/Kliniker-enheter/ Onkologiskt-centrum/Vardprogram-Kvalitetsregister/

35. Bergkvist L, Frisell J, Liljegren G et al (2001) Multicentre study of detection and false-negative rates in sentinel node biopsy for breast cancer. Br J Surg 88:1644-1648

36. Bergkvist L, Frisell J (2005) Multicentre validation study of sentinel node biopsy for staging in breast cancer. Br J Surg 92:1221-1224 (Swedish Breast Cancer Group; Swedish Society of Breast Surgeons)

37. Canadian Cancer Society/National Cancer Institute of Canada (2006) Current incidence and mortality in "Canadian cancer statistics 2006". Canadian Cancer Society/National Cancer Institute of Canada, Toronto, Canada

38. Canadian Cancer Society/National Cancer Institute of Canada (2007) Current incidence and mortality in "Canadian cancer statistics 2007". Canadian Cancer Society/National Cancer Institute of Canada, Toronto, Canada

39. Berry D, Cronin K, Plevritis S et al (2005) Effect of screening and adjuvant therapy on mortality from breast cancer. $\mathrm{N}$ Engl $\mathrm{J}$ Med 353:1784-1792

40. Ugnat AM, Xie L, Semenciw R et al (2005) Survival patterns for the top four cancers in Canada: the effects of age, region and period. Eur J Cancer Prev 14:91-100

41. Neutel CI, Gao RN, Wai E et al (2005) Trends in in-patient hospital utilization and surgical procedures for breast, prostate, lung and colorectal cancers in Canada. Cancer Causes Control 16:1261-1270

42. Gaudette LA, Gao RN, Spence A et al (2004) Declining use of mastectomy for invasive breast cancer in Canada, 1981-2000. Can J Public Health 95:336-340

43. Shen N, Mayo N, Scott S et al (2003) Factors associated with pattern of care before surgery for breast cancer in Quebec between 1992 and 1997. Med Care 41:1353-1366

44. Porter GA, McMulkin H, Lovrics PJ (2003) Sentinel lymph node biopsy in breast cancer: Canadian practice patterns. Ann Surg Oncol 10:255-260

45. SR JemalA, Ward E et al (2006) Cancer statistics. CA Cancer J Clin 56:106-130

46. Surveillance epidemiology and end results (SEER) database cancer fact sheets: cancer of the breast. SEER Database. http:// seer.cancer.gov/statfacts/html/breast.html?statfacts_page=breast. $\mathrm{html} \& \mathrm{x}=14 \& \mathrm{y}=16.2007$

47. McCracken M, Olsen M, Chen MS et al (2007) Cancer incidence, mortality, and associated risk factors among Asian Americans of Chinese, Filipino, Vietnamese, Korean, and Japanese ethnicities. CA Cancer J Clin 57:190-205

48. Sariego J (2009) Patterns of breast cancer presentation in the United States: does geography matter? Am Surg 75:545-550
49. Anderson LM, May DS (1995) Has the use of cervical, breast, and colorectal cancer screening increased in the United States? Am J Public Health 85:840-842

50. Laughlin CodyHS, EH TrilloC et al (1991) Have changing treatment patterns affected outcome for operable breast cancer? Ten-year follow-up in 1288 patients, 1965-1978. Ann Surg 213:297-307

51. Schwartz GF, Giuliano AE, Veronesi U (2002) Proceedings of the consensus conference on the role of sentinel lymph node biopsy in carcinoma of the breast, April 19-22, 2001, Philadelphia, Pennsylvania. Cancer 94:2542-2551

52. Giuliano E (2003) Sentinel node biopsy: standard of care. Breast J 9:S3-S6

53. Edge SB, Niland JC, Bookman MA et al (2003) Emergence of sentinel node biopsy in breast cancer as standard-of-care in academic comprehensive cancer centers. J Natl Cancer Inst 95:1514-1521

54. Ward E, Jemal A, Cokkinides V et al (2004) Cancer disparities by race/ethnicity and socioeconomic status. CA Cancer J Clin 54:78-93

55. Jemal A, Siegel R, Ward E et al (2007) Cancer statistics, 2007. CA Cancer J Clin 57:43-66

56. Tajima T (2006) Toward optimized breast cancer care with East/ West-linked wisdom. Breast J 12:S126-S127

57. Bray F, McCarron P, Parkin DM (2004) The changing global patterns of female breast cancer incidence and mortality. Breast Cancer Res 6:229-239

58. Ahn SH, Yoo KY (2006) Chronological changes of clinical characteristics in 31, 115 new breast cancer patients among Koreans during 1996-2004. Breast Cancer Res Treat 99:209-214

59. Yasui Y, Potter JD (1999) The shape of age-incidence curves of female breast cancer by hormone-receptor status. Cancer Causes Control 10:431-437

60. Clemmesen J (1948) The Danish cancer registry: problems and results. Acta Pathol Microbiol Scand 25:26-33

61. Anderson WF, Chen BE, Jatoi I et al (2006) Effects of estrogen receptor expression and histopathology on annual hazard rates of death from breast cancer. Breast Cancer Res Treat 100:121-126

62. Anderson WF, Pfeiffer RM, Dores GM et al (2006) Comparison of age distribution patterns for different histopathologic types of breast carcinoma. Cancer Epidemiol Biomarkers Prev 15:1899_ 1905

63. Perou CM, Sorlie T, Eisen MB et al (2000) Molecular portraits of human breast tumours. Nature 406(6797):747-752

64. Lawson JS, Field AS, Champion S et al (1999) Low oestrogen receptor alpha expression in normal breast tissue underlies low breast cancer incidence in Japan. Lancet 354(9192):1787-1788

65. Nagata C, Matsubara T, Fujita $\mathrm{H}$ et al (2005) Mammographic density and the risk of breast cancer in Japanese women. Br J Cancer 92:2102-2106

66. del Carmen MG, Halpern EF, Kopans DB et al (2007) Mammographic breast density and race. AJR Am J Roentgenol 188:1147-1150

67. El-Bastawissi AY, White E, Mandelson MT et al (2001) Variation in mammographic breast density by race. Ann Epidemiol $11: 257-263$

68. Ursin G, Ma H, Wu AH et al (2003) Mammographic density and breast cancer in three ethnic groups. Cancer Epidemiol Biomarkers Prev 12:332-338

69. Matsuno RK, Anderson WF, Yamamoto S et al (2007) Early- and late-onset breast cancer types among women in the United States and Japan. Cancer Epidemiol Biomarkers Prev 16:1437-1442

70. Leung GM, Thach TQ, Lam TH et al (2002) Trends in breast cancer incidence in Hong Kong between 1973 and 1999: an ageperiod-cohort analysis. Br J Cancer 87:982-988 
71. Sim X, Ali RA, Wedren S et al (2006) Ethnic differences in the time trend of female breast cancer incidence: Singapore, 19682002. BMC Cancer 6:261

72. Chu KC, Anderson WF, Fritz A et al (2001) Frequency distributions of breast cancer characteristics classified by estrogen receptor and progesterone receptor status for eight racial/ethnic groups. Cancer 92:37-45

73. Porter PL (2009) Global trends in breast cancer incidence and mortality. Salud Publica Mex 51(Suppl 2):s141-s146

74. Setiawan VW, Monroe KR, Wilkens LR et al (2009) Breast cancer risk factors defined by estrogen and progesterone receptor status: the multiethnic cohort study. Am J Epidemiol 16:12511259

75. Chen WY, Colditz GA (2007) Risk factors and hormone-receptor status: epidemiology, risk-prediction models and treatment implications for breast cancer. Nat Clin Pract Oncol 4:415-423

76. Pike MC, Kolonel LN, Henderson BE et al (2002) Breast cancer in a multiethnic cohort in Hawaii and Los Angeles: risk factoradjusted incidence in Japanese equals and in Hawaiians exceeds that in whites. Cancer Epidemiol Biomarkers Prev 11:795-800 